\title{
High-normal thyroid-stimulating hormone in euthyroid subjects is associated with risk of mortality and composite disease endpoint only in women
}

\author{
Servet Altay ${ }^{1}$, Altan Onat ${ }^{2}$, Günay Can $^{3}$, Eyyup Tusun ${ }^{4}$, Barış Şimşek ${ }^{5}$, Adnan Kaya ${ }^{6}$
}

\author{
1'Department of Cardiology, Trakya University, Edirne, Turkey \\ 2Department of Cardiology, Cerrahpaşa Medical Faculty, Istanbul University, Istanbul, \\ Turkey \\ ${ }^{3}$ Department of Public Health, Cerrahpaşa Medical Faculty, Istanbul University, \\ Istanbul, Turkey \\ ${ }^{4}$ Department of Cardiology, Sanliurfa Education and Research Hospital, Şanlıurfa, \\ Turkey \\ ${ }^{5}$ Department of Cardiology, Siyami Ersek Center for Cardiovascular Surgery, Istanbul, \\ Turkey \\ ${ }^{6}$ Department of Cardiology, Suruç State Hospital, Şanliurfa, Turkey
}

Submitted: 10 June 2016

Accepted: 17 September 2016

Arch Med Sci 2018; 14, 6: 1394-1403

DOI: https://doi.org/10.5114/aoms.2016.63264

Copyright @ 2016 Termedia \& Banach

\section{Abstract}

Introduction: The aim of the study was to evaluate whether serum thyroid-stimulating hormone (TSH) within the normal range in euthyroid subjects (having normal free triiodothyronine (fT3) and thyroxine (fT4)) is related to the risk of overall mortality or a composite endpoint of death and nonfatal events. Material and methods: In 614 middle-aged adult hospital screenees, free of uncontrolled diabetes at baseline, the association of sex-specific TSH tertiles with death was prospectively assessed using Cox regression, with the composite endpoint assessed using logistic regression in adjusted analyses, stratified by gender.

Results: In total, 64 deaths and additional incident nonfatal events in 141 cases were recorded at a mean 7.55 years' follow-up. Multivariable linear regression revealed TSH to be significantly associated among men with age ( $p=$ 0.006 ), but in women inversely with fT3 and fT4 ( $p<0.001$, and $p=0.024$ respectively). In logistic regression analysis, adjusted for age, fT3, fT4, systolic blood pressure and serum total cholesterol, sex-specific baseline TSH tertiles were associated in men neither with the risk of death nor with composite endpoint. In contrast, in women, the highest compared with the bottom TSH tertile predicted the risk of composite endpoint (relative risk: 2.02 , 95\% Cl: 1.07-3.82) and, much more strongly, the mortality risk, independently of fT4 increments. Conclusions: The significant association of higher range of normal serum TSH in euthyroid middle-aged adults with the risk of death and nonfatal adverse outcomes in women alone cannot be accounted for by the action of thyroid hormone and is consistent with involvement of TSH in the pro-inflammatory state.

Key words: cardiovascular events, euthyroid status, mortality, proinflammatory state, thyroid hormones, thyroid-stimulating hormone.

\section{Introduction}

It remains unclear whether normal thyroid function in euthyroid status affects clinical outcomes adversely. Though association of a single

\author{
Corresponding author: \\ Asst. Prof. Servet Altay \\ Sukrupasa mah. \\ Namik Kemal cad. 4B blok \\ No: 17, Edirne, Turkey \\ Phone: +90 5063779751 \\ E-mail: svtaltay@gmail.com
}


measurement of low thyroid-stimulating hormone (TSH) with all-cause and cardiovascular mortality in elderly individuals has been reported [1], it is also recognized that most subjects at risk for cardiovascular disease are euthyroid in the clinical setting. Population-based studies evaluating the risk of clinical outcomes in individuals with normal thyrotropin levels within the reference range have been controversial. The HUNT study reported positive and linear associations of thyrotropin levels within the reference range with coronary heart disease (CHD) mortality in Norwegian women but not men [2]. It remains unclear whether and how thyroid hormone levels within the normal range modulate mortality in euthyroid individuals.

We demonstrated for the first time in a general population that enhanced pro-inflammatory state associated with high-density lipoprotein (HDL) dysfunction is a prominent feature of the cardiometabolic risk profile among Turkish adults [3] who are prone to the metabolic syndrome (MetS). In this population, high serum apoA-I levels conferred risk of type-2 diabetes [4]. Epidemiological evidence derived from the cohort of the Turkish Adult Risk Factor (TARF) study further demonstrated that, in a milieu of enhanced inflammation, serum proteins such as lipoprotein $(\operatorname{Lp})(\mathrm{a})[5,6]$, creatinine [7-9], or asymmetric dimethylarginine [10] are involved in immune processes which lead to increased cardiometabolic risk [11]. Collectively, these observations indicated that multiple circulating polypeptide/proteins may sustain epitope damage in persons under oxidative stress and thereby serve in conferring morbidity or mortality.

Moreover, we have recently reported that low TSH levels within the normal range $(0.3$ to $0.83 \mathrm{mIU} / \mathrm{l})$ in middle-aged men - but not women - may be independent predictors of incident CHD [12]. Autoimmune responses involving serum $L p(a)$ under oxidative stress were considered to be implicated mechanistically. Similarly, low levels of thyrotropin were found to be associated with increased risk of all-cause mortality among Israeli adults related to a health service [13].

We, therefore, explored in longitudinal analyses whether levels within the normal range of TSH a circulating protein - among euthyroid applicants of a tertiary hospital might be related to all-cause mortality or to a composite endpoint consisting of nonfatal and fatal cardiac and non-cardiac events.

\section{Material and methods}

\section{Study sample}

The baseline study sample was formed by 614 outpatients and admitted patients, aged 40 to 79 years, who applied in the first 3 months of the year 2008 to the Siyami Ersek Center for Cardiovascular Surgery and had available normal values for TSH (0.34-5.6 mlU/l), fT3 $(2.3-4 \mathrm{pg} / \mathrm{ml})$ and fT4 (0.59-1.64 ng/dl) concentrations. This sample was derived from attendees of the center in the stated period totaling 2338 patients after exclusion of subjects using thyroid medication or having a history of thyroid disease, renal failure (eGFR $\left.<30 \mathrm{ml} / \mathrm{min} / 1.73 \mathrm{~m}^{2}\right)$, acute coronary syndrome or myocardial infarction, uncontrolled diabetes $\left(\mathrm{HbA}_{1 c}>10 \%\right)$, decompensated heart failure, stroke, active infection, malignancy, or chronic obstructive pulmonary disease (COPD). From this group, patients with missing values of thyroid hormones, patients having thyroid hormone values outside the indicated reference values, examined outpatients with missing other laboratory values and subjects with no follow-up were also excluded. None of these patients had undergone cardiovascular surgery. Patients included with baseline diabetes under fair control were 130 in number. Follow-up duration was up to 8 years. The study was approved by the Institutional Ethics Committee of the stated center. Data recorded in the hospital charts were obtained. The study was carried out according to the principles of the Declaration of Helsinki.

\section{Measurement of risk factors and validation}

Blood pressure (BP) was measured twice in the sitting position on the right arm using a sphygmomanometer (Erka), after at least 5 min of rest. The mean of two recordings 3 min apart was computed. Body mass index (BMI) was computed from values of weight/height squared. Never, former and current smokers formed the categories of cigarette smoking.

Blood samples, collected after an overnight fast and uniformly between 8 and 10 a.m., were analyzed in the hospital central laboratory, using Siemens Healthcare Diagnostic Products kits and calibrators (Marburg, Germany). Serum TSH, fT4 and fT3 concentrations were measured by the electrochemiluminescence immunoassay (ECLIA) method using a commercial kit (Beckman Coulter, Brea, CA, USA). Normal values in our center's laboratory were as follows: TSH: $0.35-5.6 \mathrm{mIU} / \mathrm{l}$, fT3: 2.3-4.0 pg/ml, fT4: 0.59-1.64 ng/dl. Within-run and day-to-day coefficient of variation (CV) for TSH were $3.3 \%$ and $4.6 \%$, respectively. Serum uric acid concentrations were measured enzymatically with the uricase method, and creatinine with the Jaffe method having a lower detection limit of $0.1 \mathrm{mg} / \mathrm{dl}$. Serum concentrations of total cholesterol, triglycerides, glucose and total bilirubin were measured enzymatically. Low-density lipoprotein (LDL)- and HDL-cholesterol were quantified directly with the elimination catalase method and Advia autoanalyzer. Concentrations of $\mathrm{HbA}_{1 \mathrm{c}}$ were measured in whole blood agglutination inhibition and serum C-reactive protein (CRP) with 
latex-enhanced immunoturbidimetry. Left ventricular ejection fraction (LVEF) was calculated using the modified Simpson's method.

\section{Definitions}

Diabetes type 2 was diagnosed with the criteria of the American Diabetes Association (ADA) [14]. Coronary heart disease was diagnosed by a history of myocardial infarction or revascularization, by greater than $50 \%$ stenosis of at least one major coronary artery on angiograms.

\section{Outcome}

Information on cause of death was drawn from hospital records during follow-up, from records of the nationwide Identity Participation System, or from first-degree relatives. Chronic CHD denotes patients with coronary disease in whom greater than $50 \%$ stenosis was identified in the coronary angiogram, or percutaneous coronary intervention or coronary artery bypass surgery performed during the follow-up period. Congestive heart failure (HF) was diagnosed when typical symptoms (e.g. breathlessness, ankle swelling, and fatigue) and signs (e.g. elevated jugular venous pressure, pulmonary crackles, and displaced apex beat) resulting from an abnormality of cardiac structure were observed. Stroke was defined as affected impairment of brain function, which has resulted in an inability to move a limb, or inability to understand or formulate speech. Patients who reported commencement in the follow-up period (after the index dates) of new manifestations of a malignancy (beyond that involving the skin) were designated to have incident malignancy, and its type was recorded, when known. Incident COPD designated those patients who had no evidence of this at baseline but received this diagnosis in the follow-up period in our out-patient chest clinic, based on obstructive findings in pulmonary function testing and in whom bronchodilator drugs were initiated.

\section{Statistical analysis}

Descriptive parameters were shown as mean ( \pm standard deviation (SD)) or percentages. Due to skewed distribution, geometric means were used for TSH, triglyceride and CRP values. Two-sided $t$-tests and Pearson's $\chi^{2}$ tests were used to analyze the differences between means and proportions of multiple groups, and pairwise comparisons with post hoc Tukey HSD tests served to detect significance of values in multiple groups.

The following cutoff points defined the TSH mid-tertile in men and women, respectively: 1.061.74, and 1.329-2.32 $\mathrm{mlU} / \mathrm{l}$. The development of multiple nonfatal events or death in an individu- al was counted only as one subject, and nonfatal events identified in patients who subsequently died were not included in analyses of nonfatal events.

The composite endpoint was defined as overall deaths plus incident nonfatal events of $\mathrm{CHD}$, myocardial infarction (MI), stroke, HF, atrial fibrillation, thromboembolism, valve thrombosis, renal failure, COPD and malignancy, all of which may be a consequence of a pro-inflammatory state and autoimmune activation according to reports in the literature (Ref. [11] may be consulted). A multiple linear regression model, selected from findings of univariate correlation, was analyzed to seek the best independent covariates of TSH. Cox proportional hazard regression was used for all-cause mortality and - due to the uncertain timing of some nonfatal events - logistic regression analyses for the combined endpoint with associations of baseline TSH adjusted for age, fT3, fT4, systolic $\mathrm{BP}$ and serum total cholesterol, stratified by gender. Risk estimates (RR) and 95\% confidence intervals $(\mathrm{Cl})$ were obtained for continuous variables, expressed per 1-SD increment. A $p<0.05$ on the two-tail test was considered statistically significant. Statistical analyses were performed using SPSS-10 for Windows.

\section{Results}

Mean values (SD) for thyroid hormones in euthyroid male and female individuals, respectively, were as follows: 1.34 (1.81-fold) and 1.69 (1.89fold) $\mathrm{mIU} / \mathrm{l}$ of TSH, $3.05 \pm 0.34$ and $2.968 \pm 0.32 \mathrm{pg} /$ $\mathrm{ml}$ of $\mathrm{fT} 3 ; 0.85 \pm 0.15$ and $0.856 \pm 0.15 \mathrm{ng} / \mathrm{dl}$ of fT4 Hence, though fT 3 and fT 4 were similar in the sexes, geometric mean serum TSH was $26 \%$ higher in women than men. This is very similar to sex-specific values (23\% higher in women) in euthyroid adults in the Turkish population at large [12].

Median follow-up was 7.7 years, and mean follow-up (from the first visit in early 2008 to the last visit or telephone call in January 2016) was 7.75 \pm 0.25 years (similar in the sexes), yielding a total follow-up of 4760 person-years. The death rate was 13.4 per 1000 person-years, and the composite endpoint incidence rate was 43 per 1000 person-years.

\section{Baseline characteristics}

Characteristics at baseline of the male and female sample $(n=614)$, stratified by serum TSH tertiles, are presented in Table I. Among 18 variables tabulated, age in the upper tertile and fasting triglycerides in the lower tertile alone were in men significantly higher than in the remainder. Females in the lower tertile were older and had significantly higher fT4, and women in the upper tertile had higher CRP. 


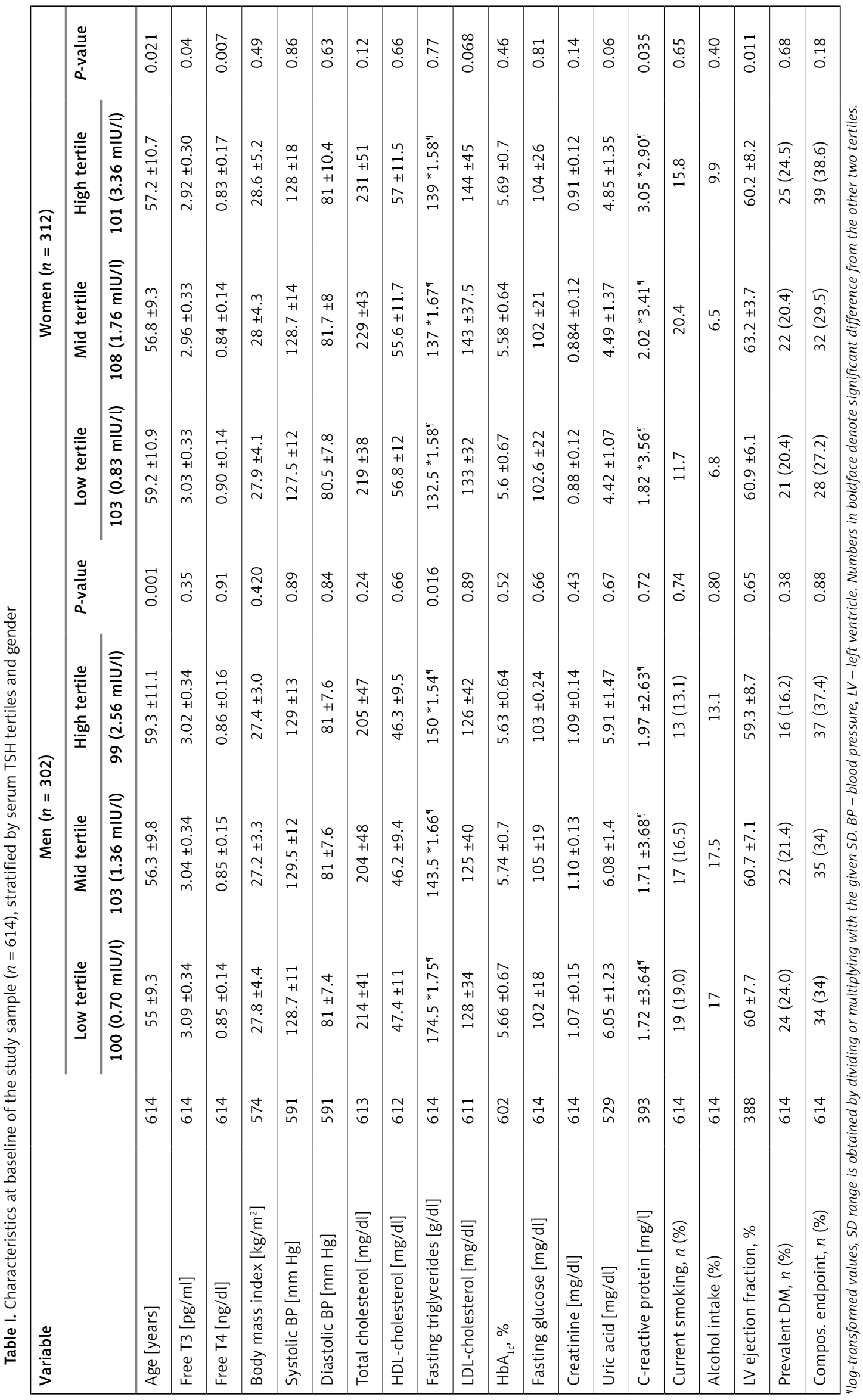




\section{Correlates and independent covariates of TSH}

Pearson correlations of 10 variables with TSH are available in Table II. Correlations were significant in men only with age and inversely with fasting triglycerides and of borderline significance with fT3. Correlations were significant in women inversely with fT3 and fT4 and positively with total and LDL-cholesterol levels.

Table III shows findings of multiple linear regression analyses in men and women with five selected covariates. Whereas log TSH was significantly associated in men only with age, it was inversely associated in women with circulating fT3 and fT4.

\section{Findings at follow-up}

Thyroid-stimulating hormone levels 5.1 to 5.6 $\mathrm{mIU} / \mathrm{l}$ existed in 7 women and 5 men alone, in whom mean fT4 averaged $0.803 \mathrm{ng} / \mathrm{dl}$ and 2 deaths occurred at follow-up. Though thyroidrelated proteins were similar in male survivors vs. deceased ones, these were significantly differ- ent in women at baseline: TSH $1.64{ }^{*} 1.88 \mathrm{mIU} / \mathrm{l}$ in survivors vs. $2.35{ }^{*} 1.87 \mathrm{mIU} / \mathrm{l}$ in subsequently deceased ones $(p=0.004)$, fT3 $2.98 \pm 0.33$ vs 2.83 $\pm 0.26 \mathrm{pg} / \mathrm{ml}(p=0.016)$, fT4 $0.85 \pm 0.14$ vs. 0.97 $\pm 0.21 \mathrm{ng} / \mathrm{dl}(p=0.007)$, respectively.

During the follow-up, 36 deaths and 70 nonfatal primary events occurred in men (total composite endpoint in 106 cases), 28 deaths and 71 nonfatal primary events occurred in women (total composite endpoint in 99 cases). The distribution of causes of death and of incident nonfatal events is given separately for men and women in Table IV.

Age-related analysis of deaths was as follows. Whereas mean \pm SD age in survivors was 56.5 \pm 10.1 years, it was $63.6 \pm 9.7$ years in subsequently deceased subjects at baseline. Cross-tabulation of deaths in TSH tertiles of each age group from 40-49 to $\geq 70$ years (not shown) demonstrated a graded increase in the frequency of deaths with increments of TSH (which proved to be significant $(p=0.032)$ in the age group $\geq 70$ years.

Table II. Pearson correlations at baseline with log thyrotropin in men and women

\begin{tabular}{|lcccc|}
\hline Parameter & \multicolumn{2}{c}{ Men $(N=302)$} & \multicolumn{2}{c|}{ Women $(N=312)$} \\
\cline { 2 - 5 } & $r$ & $P$-value & $r$ & $P$-value \\
\hline Age & 0.20 & 0.001 & -0.10 & 0.08 \\
\hline Free T3 & -0.11 & 0.056 & -0.18 & 0.002 \\
\hline Free T4 & -0.01 & 0.87 & -0.17 & 0.003 \\
\hline Systolic blood pressure & 0.02 & 0.59 & 0.05 & 0.42 \\
\hline Diastolic blood pressure & 0.04 & 0.56 & 0.02 & 0.76 \\
\hline Total cholesterol & 0.06 & 0.56 & 0.12 & 0.034 \\
\hline LDL-cholesterol & -0.01 & 0.81 & 0.12 & 0.041 \\
\hline HDL-cholesterol & 0.00 & 0.99 & 0.02 & 0.70 \\
\hline F. triglycerides" & -0.13 & 0.027 & 0.06 & 0.31 \\
\hline C-reactive protein" & 0.08 & 0.28 & 0.08 & 0.29 \\
\hline
\end{tabular}

"log-transformed. CRP measurement was available only in 391 individuals.

Table III. Multiple linear regression analysis of covariates with TSH"

\begin{tabular}{|lcccccc|}
\hline Parameter & \multicolumn{3}{c}{ Men $(N=290)$} & \multicolumn{3}{c|}{ Women $(N=309)$} \\
\cline { 2 - 7 } & Coeff. $\boldsymbol{\beta}$ & SE & $P$-value & Coeff. $\boldsymbol{\beta}$ & SE & $P$-value \\
\hline Age, 11 years & 0.044 & 0.011 & 0.006 & -0.033 & 0.022 & 0.057 \\
\hline Free triiodothyronine (fT3), $0.33 \mathrm{pg} / \mathrm{ml}$ & -0.017 & 0.015 & 0.24 & -0.057 & 0.016 & $<0.001$ \\
\hline Free thyroxine (fT4), $0.15 \mathrm{ng} / \mathrm{ml}$ & 0.01 & 0.015 & 0.52 & -0.035 & 0.015 & 0.024 \\
\hline Total cholesterol, $40 \mathrm{mg} / \mathrm{dl}$ & 0.000 & 0.04 & 0.66 & 0.04 & 0.04 & 0.49 \\
\hline LDL-cholesterol, $38 \mathrm{mg} / \mathrm{dl}$ & 0.000 & 0.038 & 0.75 & -0.0006 & 0.038 & 0.99 \\
\hline Variance; $r^{2}$ & \multicolumn{3}{c}{$3 \% ; p=0.044$} & & $8 \% ; p<0.01$ & \\
\hline
\end{tabular}

"log-transformed values. 
Table IV. Distribution of cause of deaths and nonfatal events (total $N=205$ ) in the study sample

\begin{tabular}{|c|c|c|c|c|c|c|c|}
\hline \multirow[t]{2}{*}{ Variable } & \multicolumn{3}{|c|}{ Deaths } & \multicolumn{4}{|c|}{ Nonfatal events } \\
\hline & Total & Male & Female & Overall* & Total & Male & Female \\
\hline Myocardial infarction & 12 & 10 & 2 & 18 & 12 & 9 & 3 \\
\hline Coronary heart disease & 3 & 2 & 1 & 72 & 57 & 29 & 28 \\
\hline Stroke & 4 & 1 & 3 & 0 & 0 & & \\
\hline Heart failure & 5 & 1 & 4 & 12 & 8 & 5 & 3 \\
\hline Atrial fibrillation & 0 & & & 14 & 5 & 2 & 3 \\
\hline Valve thrombosis & 1 & & 1 & & 0 & & \\
\hline Renal failure & 1 & & 1 & 11 & 4 & 3 & 1 \\
\hline Pulmonary embolism & 1 & & 1 & 11 & 0 & & \\
\hline Type 2 diabetes & 0 & & & 51 & 40 & 13 & 27 \\
\hline Sepsis & 2 & 1 & 1 & & 0 & & \\
\hline Liver cirrhosis & 1 & & 1 & & 0 & & \\
\hline Colon perforation & 1 & 1 & & & 0 & & \\
\hline C. obstr. pulmon. dis. & 3 & & 3 & 11 & 4 & 3 & 1 \\
\hline Malignancy** & 11 & 6 & 5 & 11 & 11 & 6 & 5 \\
\hline Cause indeterminate & 19 & 14 & 5 & 0 & 0 & & \\
\hline Total & 64 & 36 & 28 & 211 & 141 & 70 & 71 \\
\hline
\end{tabular}

${ }^{\star}$ Comprises 70 nonfatal events additional to death or the primary unique event. ${ }^{* *} / n c i d e n t ~ 10$ malignancies in women: 4 breast, 2 lung,

1 pancreas, 3 type unknown. Among 12 malignancies in men: 4 lung, 1 colon, 1 prostate, 1 renal cell carcinoma, 5 type unknown.

\section{Predictors of mortality risk}

Table $V$ provides sex-specific findings using Cox regression models for the risk of all-cause mortality. In men TSH tertiles were not significantly associated in either of the two models, and only fT4 was so in the fully adjusted model. In contrast, in women, independently of age and fT4 levels, the TSH top tertile was almost 10-fold (95\% Cl: 2.50-39.1) more strongly associated with death risk compared to the lowest tertile, as was $p$-trend significant. Further addition of fasting glucose to the model modestly strengthened in women the predictive ability of both the upper TSH tertile and fT4 (not shown in Table V). In regard to logistic regression analyses for the composite endpoint, no parameter, apart from age, was related in males, while in women, other than age and at borderline significance fT4, upper compared with the lowest TSH tertile predicted the risk of composite endpoint (relative risk: 2.02, 95\% Cl: 1.07-3.82).

\section{Discussion}

In this longitudinal study in middle-aged euthyroid Turkish adults (derived from hospital screenees) who had serum TSH within the normal range, we sought a possible predictive value for mortality or for a composite index of death and nonfatal cardiac and non-cardiac events. Key findings were: a) a clear gender disparity, men showing expectedly no association with tertiles of TSH, in contrast to women; b) the upper TSH tertile in women revealed somewhat surprisingly strong predictive ability of the death risk and a significant 2-fold risk of the composite endpoint compared with the lowest tertile, independently of circulating fT4 and other potential confounders. We also documented that TSH in euthyroid men was not associated with the thyroid hormone levels, while in euthyroid women a significant inverse association existed with such levels. These findings led to theconclusion that pro-inflammatory involvement of the upper normal TSH tertile in women likely mediated the adverse outcomes (explained below).

\section{Normal range of thyroid proteins}

Laboratory reference values used for TSH were identical to those reported by Laclaustra et al. [15], similar to those reported by Zhang et al. [16] (0.28-5.0 $\mathrm{mIU} / \mathrm{l})$, for US people by Rhee et al. [17] (0.5-5.0 $\mathrm{mlU} / \mathrm{l})$, or for Israelis [13] (0.35-4.2 $\mathrm{mlU} / \mathrm{l})$, though being wider than those reported in the HUNT study [2]. Reference ranges have been con- 


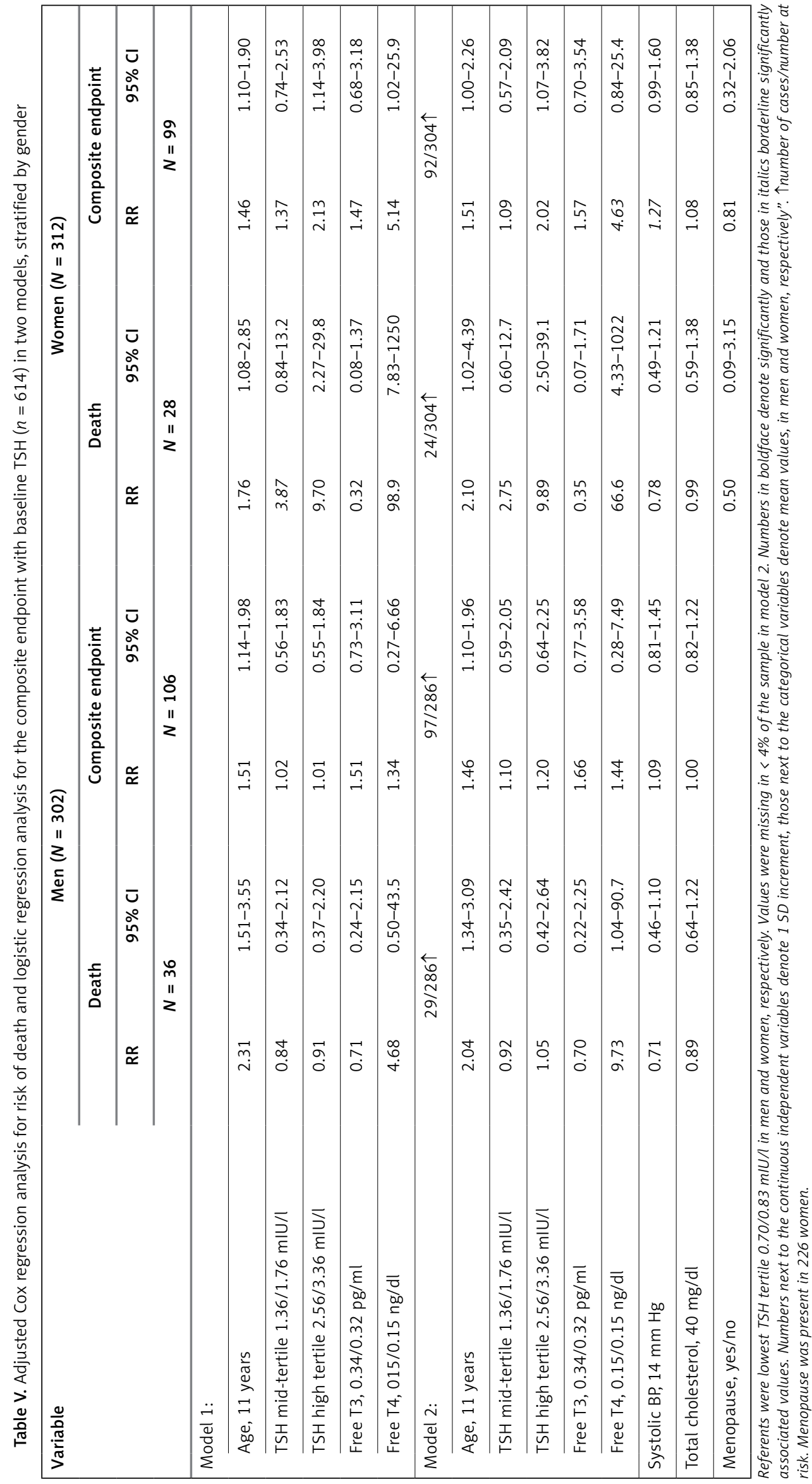


sidered to possibly differ in different races, populations or ages [18], but they were clearly not sufficiently widely different herein to substantially affect the analysis of outcomes.

\section{Previous reports}

In the recent HUNT study, using hospital-based diagnosis of myocardial infarction, 556 died from CHD. High TSH within the reference range was associated with increased risk of coronary mortality in women, but not men [19]. Yet thyroid function was not associated with the risk of being hospitalized with MI. This suggests that high-normal TSH values in women seem to contribute to death more than to chronic CHD. The current findings corroborate this and extend to mortality and diseases other than CHD.

Thyroid-stimulating hormone levels within the normal range were not associated with overall or cardiovascular mortality among northeastern Germans [20]. In Dutch patients with manifest vascular diseases, recurrent cardiovascular events and mortality were not predicted by TSH as well [21].

The association between thyroid hormones and mortality in euthyroid individuals was recently studied in over 210,000 young and middle-aged Korean adults undergoing health-screening examination in a hospital. Subjects in the lowest fT4 quartile were associated with the risk of allcause mortality, and those in the lowest fT4 quartile were associated with (especially liver) cancer mortality at a 4-year follow-up [16]. Thyroid-stimulating hormone levels were not associated with mortality endpoints. Our results were distinctly different inasmuch as the high TSH tertile predicting all-cause mortality was accompanied by lower fT4 and fT3 values and an independent inverse association of TSH with the hormone levels was determined. Moreover, higher increments of fT4 predicted the endpoints. The disparity may partly be due to the relatively young age in the Korean study, as it has been suggested that age might be one factor explaining the discrepancies between the various reported studies [18].

Similar to current results, a high-normal TSH (but not a low-normal fT4) was associated with mortality in the cross-sectional Nijmegen Biomedical Study in the elderly Dutch [21]. It is well possible that a level of chronic low-grade inflammation was attained among Dutch people at an older age than in Turks. Boggio et al. [22] investigated cross-sectionally in 744 euthyroid Italian women the association of TSH within the normal reference range with the MetS, the prevalence of which was significantly higher in women with TSH above than below the median value $(2.1 \mathrm{lU} / \mathrm{ml})$. Age-adjusted logistic regression analysis confirmed such an association with the MetS $(O R=1.38)$.
In 8010 German patients with CHD undergoing percutaneous coronary intervention, the upper TSH tertile within the reference range was associated with significant 1.31-fold higher 3-year mortality per each tertile increase [23]. Similarly, the upper-normal (> 3.0 to $5.0 \mathrm{mIU} / \mathrm{l}$ ) TSH levels were associated with higher adjusted death risk in time-dependent analyses also in a U.S. national cohort of incident hemodialysis patients [17]. The stated increased death risk paralleled clinical hypothyroidism.

A gender difference in elderly euthyroid Israelis in low-normal thyrotropin levels was reported to be associated with increased risk of hip fractures [24]. In addition to gender dependence, it is remarkable that both high-normal TSH [17, $19,23,24]$ and low-normal TSH levels $[12,24]$ in euthyroid individuals predict risk of adverse outcomes, including mortality. This is suggestive of pro-inflammatory state and autoimmune activation affecting (often in one gender) the circulating protein TSH and driving the risk of adverse outcomes, as further detailed below. An analogy may be made to pro-inflammatory/dysfunctional HDL, which proved to be significantly correlated with carotid intima media thickness in middle-aged South Asian immigrants in the US [25].

The current finding of an association between increments of fT4 in the normal range and death/ composite endpoint is essentially in line with a cross-sectional case-control study among Koreans [26], in which higher serum fT4 levels even within the normal range were associated with coronary artery disease, warranting further investigation.

In a subgroup of the Framingham study on biochemically euthyroid individuals, higher TSH and lower fT4 concentrations within the reference range were associated with more adverse CVD risk factors and greater indication of body accumulation on cross-sectional analysis, whereas at 6-year follow-up thyroid hormones were not associated [27]. Yet in the longitudinal analysis individuals with diverse major cardiovascular risk factors at baseline were excluded.

The predictive ability of high-normal TSH values (tertile 3) for the composite endpoint was accompanied by increments of circulating fT4 in the normal range, independent of age, systolic BP and total cholesterol concentrations. Optimal thyroid hormone levels in older people have been questioned in the recent Cardiovascular Health Study [28], which found that higher fT4 levels were associated with higher incidence of atrial fibrillation, CHD, heart failure and mortality. However, mortality was predicted by decrements of TSH [29], in contrast to the current findings in women. The possibility of borderline subclinical hyperthyroidism may be discarded, given the finding herein that higher TSH was associated with low- 
er fT4 concentrations (and positively correlated with total cholesterol values) that predicted the composite endpoint. The current findings do not indicate that high-normal levels of TSH and fT4 occur in the same female subjects. Indeed, these were modestly though significantly independently inversely associated. This stated, it is still conceivable that the feedback mechanism between the two hormones may have been disrupted in some study participants with high-normal TSH levels.

\section{Possible mechanism of the prediction of adverse outcomes by high-normal TSH in euthyroid women}

The discrepant findings across studies in euthyroid people may be due to varying prevalence and intensity of the pro-inflammatory state among investigated study samples, which may further be sex-dependent. Thyroid hormone levels were inversely correlated with chronic inflammation, a critical component of tumor progression [29].

The observation that in the same study sample no association existed among men of baseline TSH tertiles either with the risk of death or with that of the composite endpoint indicates that a special milieu of the pro-inflammatory state prevailed among women, a repeatedly detected and reported state among middle-aged and elderly Turkish adults [3, 6, 8, 10, 30].

In contrast to the significant inverse correlation of TSH with triglycerides in men, women exhibit a weak positive correlation, added to an inverse association with age; and the high TSH tertile in women exhibited significantly higher CRP concentrations, which collectively suggest a link to chronic enhanced systemic inflammation. It may be considered that pro-inflammatory turned TSH protein following epitope damage in duced by oxidative stress might be cleared more slowly from the circulation, and such a characteristic might explain the concomitantly increased risk of, particularly, fatal and nonfatal events. Females in the high-normal TSH tertile exhibited a significant inverse association with fT3. This is in line with the report that, among hospitalized Turkish patients with myocardial infarction, euthyroid patients with reduced fT3 levels showed significant excess mortality and major adverse cardiac events at short-term follow-up, after adjustment for confounders [31].

Implications: The finding that high-normal TSH levels in euthyroid middle-aged and elderly people are associated with excess mortality and morbidity should in similar settings alert the clinician to seek other markers of the pro-inflammatory state and to undertake protective measures, including those for avoidance of constipation, often a factor enhancing inflammation.
Strengths and limitations: Euthyroid status could be accurately determined in this study as - apart from TSH - fT4 and fT3 were measured as well, which likely applies to a minority of similar prospective studies. The length of follow-up can be considered as adequate. Existence of major gender difference in the predictive ability of outcomes shown by high-normal TSH more strongly reflects a differing underlying mechanism. The relatively low number of endpoint events is evidently a limitation which, however, was not so critical as to preclude the emergence of robust associations. The findings may have little applicability to populations or population segments not prone to MetS or impaired glucose tolerance.

In conclusion, among middle-aged and elderly euthyroid Turkish adults, high-normal TSH levels were significantly and independently associated with all-cause mortality and a composite endpoint of cardiac and non-cardiac disorders in women alone. High-normal fT4 in women additively predicted the outcome endpoints. Further studies are warranted to confirm the present findings in larger studies in different ethnicities, age groups and population segments prone to impaired glucose tolerance.

\section{Conflict of interest}

The authors declare no conflict of interest.

\section{References}

1. Parle JV, Maisonneuve P, Sheppard MC, Boyle P, Franklyn JA. Prediction of all-cause and cardiovascular mortality in the elderly people from one low serum thyrotropin result: a 10-year cohort study. Lancet 2001; 358: 861-65.

2. Åsvold BO, Bjøro T, Nilsen TI, Gunnell D, Vatten LJ. Thyrotropin levels and risk of fatal coronary heart disease. Arch Intern Med 2008; 168: 855-60.

3. Onat A, Hergenç G. Low-grade inflammation and dysfunction of high-density lipoprotein and its apolipoproteins as a major driver of cardiometabolic risk. Metabolism 2011; 60: 499-512.

4. Onat $A$, Hergenç G, Bulur S, Uğur $M$, Küçükdurmaz $Z$, Can G. The paradox of high apolipoprotein A-l levels independently predicting incident type-2 diabetes among Turks. Int J Cardiol 2010; 142: 72-9.

5. Onat A, Can G, Murat S, Çiçek G, Örnek E, Yüksel H. Aggregation of lipoprotein(a) to apolipoprotein A-I underlying HDL dysfunction as a major coronary risk factor. Anadolu Kardiyol Derg 2013; 13: 543-51.

6. Onat A, Çoban N, Can G, et al. Low “quotient” Lp(a) concentration mediating autoimmune activation predicts cardiometabolic risk. Exp Clin Endocr Diabetes 2014; 122: 11-8.

7. Onat A, Can G, Ademoğlu E, Çelik E, Karagöz A, Örnek E. Coronary disease risk curve of serum creatinine is linear in Turkish men, U-shaped in women. J Investig Med 2013; 61: 27-33.

8. Onat A, Yüksel H, Can G, Köroğlu B, Kaya A, Altay S. Serum creatinine is associated with coronary disease risk 
even in the absence of metabolic disorders. Scand J Clin Lab Inv 2013; 73: 569-75.

9. Altay S, Onat A, Özpamuk-Karadeniz F, Karadeniz Y, Kemaloğlu-Öz T, Can G. Renal "hyperfiltrators" are at elevated risk of death and chronic diseases. BMC Nephrol 2014; 15: 160

10. Onat A, Köroğlu B, Can G, Karagöz A, Çelik E, Aydın M. Apparently "low" serum asymmetric dimethylarginine is associated with fasting glucose and tends toward association with type-2 diabetes. Anadolu Kardiyol Derg 2014; 14: 26-33.

11. Onat A, Can G. Enhanced pro-inflammatory state and autoimmune activation: a breakthrough to understanding chronic diseases. Curr Pharm Design 2014; 20: 575-84.

12. Onat A, Aydın M, Can G, et al. Normal thyroid-stimulating hormone levels, autoimmune activation, and coronary heart disease risk. Endocrine 2014; 48: 218-26.

13. Pereg D, Tirosh A, Elis A, et al. Mortality and coronary heart disease in euthyroid patients. Am J Med 2012; 125: 826e7-13.

14. American Diabetes Association. Diagnosis and classification of diabetes mellitus. Diabetes Care 2012 35 (Suppl 1): S64-71.

15. Laclaustra M, Hurtado-Roca Y, Sendin M, et al. Lowernormal TSH is associated with better metabolic risk factors: a cross-sectional study on Spanish men. Nutr Metab Cardiovasc Dis 2015; 25: 1095-103.

16. Zhang Y, Chang Y, Ryu S, et al. Thyroid hormones and mortality risk in euthyroid individuals: the Kangbuk Samsung Health Study. J Clin Endocrinol Metab 2014; 99: 2467-76.

17. Rhee CM, Kim S, Gillen DL, et al. Association of thyroid functional disease. J Clin Endocrinol Metab 2015; 100: 1386-95.

18. Van de Ven AC, Netea-Maier RT, de Vegt F, et al. Associations between thyroid function and age. Eur J Endocrinol 2014; 171: 183-91.

19. Åsvold BO, Bjøro T, Platou C, Vatten LJ. Thyroid function and the risk of coronary heart disease: 12-year follow-up of the HUNT study in Norway. Clin Endocrinol 2012; 77: 911-7.

20. Ittermann T, Haring R, Sauer S, et al. Decreased serum TSH levels are not associated with mortality in the adult northeast German population. Eur J Endocrinol 2010; 162: 579-85.

21. Westerink J, van der Graaf Y, Faber DL, et al. Relation between thyroid-stimulating hormone and the occurrence of cardiovascular events and mortality in patients with manifest vascular diseases. Eur J Prev Cardiol 2012; 19: 864-73.

22. Boggio A, Muzio F, Fiscella M, Sommariva D, Branchi A. Is thyroid-stimulating hormone within the normal reference range a risk factor for atherosclerosis in women? Intern Emerg Med 2014; 9: 51-7.

23. Ndrepepa G, Braun S, Mayer K, et al. Prognostic value of thyroid-stimulating hormone within reference range in patients with coronary artery disease. Metabolism 2015; 64: 1308-15.

24. Leader A, Ayzenfeld RH, Lishner M, et al. Thyroteopin levels within the lower normal range are associated with an increased risk of hip fractures in euthyroid women, but not men, over the age of 65 years. I Clin Endocrinol Metab 2014; 99: 2665-72.

25. Dodani S, Dong L, Guirgis FW, Reddy ST. Carotid intima media thickness and low high-density lipoprotein (HDL) in South Asian immigrants: could dysfunctional HDL be the missing link? Arch Med Sci 2014; 10: 870-9.
26. Jung $\mathrm{CH}$, Rhee EJ, Shin HS, et al. Higher serum free thyroxine levels are associated with coronary artery disease. Endocrine J 2008; 55: 819-26.

27. Lee JJ, Pedley A, Marqusee E, et al. Thyroid function and cardiovascular disease risk factors in euthyroid adults: a cross-sectional and longitudinal study. Clin Endocrinol (Oxf) 2016 Jun 3. doi: 10.1111/cen.13124.

28. Cappola AR, Arnold AM, Wulczyn K, et al. Thyroid function in the euthyroid range and adverse outcomes in older adults. J Clin Endocrinol Metab 2015; 100: 1088-96.

29. Türemen EE, Çetinarslan B, Şahin T, et al. Endothelial dysfunction and low-grade chronic inflammation in subclinical hypothyroidism due to autoimmune thyroiditis. Endocrine J 2011; 58: 349-54.

30. Onat A, Karadeniz Y, Tusun E, Kaya A. Advances in understanding gender difference in cardiometabolic disease risk. Expert Rev Cardiovasc Ther 2016; 14: 513-23.

31. Özcan KS, Osmonov D, Toprak E, et al. Sick euthyroid syndrome is associated with ST segment elevation myocardial infarction undergoing primary percutaneous intervention. Cardiology J 2014; 21: 238-44. 